\title{
What are the Current Problems and Major Challenges of the Care for
}

\section{Periodontal Health}

\author{
Musurlieva N and Stoykova $\mathbf{M}^{*}$ \\ Department of Public Health, MU-Plovdiv, Bulgaria
}

*Corresponding author: Mariya Stoykova Bratoycheva, Department of Public Health, MU-Plovdiv, Bulgaria, Email: brami@abv.bg

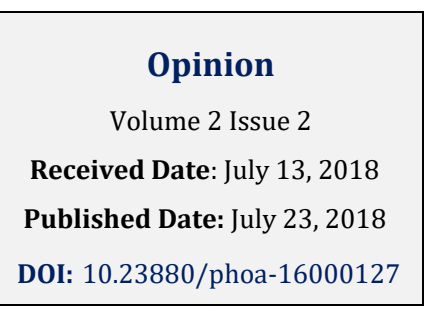

factors with chronic noncommunicable diseases, including lifestyle factors, e.g. smoking and alcohol consumption; metabolic factors, e.g. diabetes, obesity and metabolic syndrome; dietary factors and stress.

A number of studies show that periodontitis influence the quality of life (QoS) of patients. It affects negatively the physical, functional and psychological well-being, as well as the social activity of individuals. The more severe stages of periodontitis, associated with greater depth of the periodontal pockets, mobility and displacement of teeth, have a greater negative impact.

\section{What are the Current Problems and Major Challenges of the Care for Periodontal Health?}

Periodontal diseases are truly a 'silent' global epidemic with a huge disease burden and socio-economic effects. Unfortunately, awareness of periodontal health remains low worldwide, and the majority of people affected do not initiate early care, due to various personal, cultural and socio-economic factors.

One reason for the low awareness among the general public may be due to the fact that oral health and oral care are not part of the healthy lifestyle recommended by the World Health Organization (WHO). It is also noted that periodontal health is not reflected in national health policies or guidelines that mainly focus on targeting chronic noncommunicable diseases. In general, periodontal health is not perceived as a health issue, but rather a 'cosmetic' problem by many individuals and patients, due to various reasons. Patients may believe that their missing teeth can effectively be replaced and reconstructed by dental implants but this procedure poses an increased risk for the development of periimplantitis especially in periodontally susceptible individuals. 
A number of studies have reported that service availability and economic barriers are the major causes of periodontal health inequalities in the world. It should also be highlighted that oral care is usually not part of public health services, and access to professional care is difficult (especially for people with low income).

What Recommendations Can Be Made To Improve Population Periodontal Health?

Periodontal health literacy should be improved. The ability to build periodontal literacy is essential for proactive awareness of periodontal health and disease prevention among the public and dental professional communities. The link between oral diseases and general diseases should be communicated intensively to the medical profession to establish an interdisciplinary team to better manage the individuals already affected by periodontal disease. More efforts should be made to raise the awareness of periodontal health and appropriate care among general dental practitioners and other healthcare professionals.

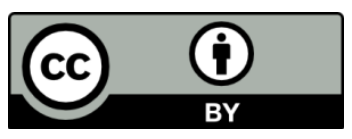

